\title{
Caractéristiques physiques et nutritionnelles des blocs multi nutritionnels fabriqués à partir des sous-produits agricoles et agroindustriels du Bénin
}

\author{
M. MONTCHO ${ }^{1}$, S. BABATOUNDE ${ }^{1 *}$, A.B. ABOH ${ }^{2}$, M.J.D. BAHINI ${ }^{1}$, \\ C.A.A.M. CHRYSOSTOME ${ }^{3}$ et G.A. MENSAH ${ }^{4}$ \\ ${ }^{I}$ Laboratoire de Zootechnie (LZ), Ecole des Sciences et Techniques de Production Animale (ESTPA), Faculté \\ des Sciences Agronomiques (FSA), Université d'Abomey-Calavi (UAC), \\ FSA/UAC, 01 BP 526 Recette principale, Cotonou, Bénin. \\ ${ }^{2}$ Ecole d'Aquaculture de la Vallée, Université d'Agriculture de Kétou (UAK), BP 43 Kétou, Bénin. \\ ${ }^{3}$ Laboratoire de Recherche Avicole et de Zoo-Economie, Ecole des Sciences et Techniques de Production \\ Animale (ESTPA), Faculté des Sciences Agronomiques (FSA), Université d'Abomey-Calavi (UAC), \\ FSA/UAC, 01 BP 526 Recette principale, Cotonou, Bénin. \\ ${ }^{4}$ Centre de Recherches Agricoles d'Agonkanmey (CRA-AgonKanmey), Institut National des Recherches \\ Agricoles du Bénin (INRAB), INRAB/01 BP 2359 Recette Principale, Cotonou République du Bénin. \\ *Auteur correspondant ; E-mail : babatoundesev@yahoo.fr ; Tél : (0) 22997446701.
}

\section{RESUME}

Au Bénin, les sous-produits considérés isolément présentent des déficiences nutritionnelles ou des caractéristiques physiques qui contrarient leur utilisation. Les blocs multi-nutritionnels (BMN), restent un moyen pour corriger ces contraintes. Ce travail vise à évaluer les caractéristiques physiques et nutritionnelles des BMN fabriqués au moyen des sous-produits localement disponibles en utilisant trois types de liants (remoulage du manioc appelé "garigo", l'amidon de manioc "goman" et l'argile). La dureté a été estimée en exerçant le pouce au milieu du bloc après séchage et la cohésion en essayant de le rompre à la main avec un effort plus grand. La couleur a été appréciée par observation directe des blocs et l'odeur grâce à l'odorat. La composition chimique a été déterminée selon les méthodes officielles de l'AOAC et les valeurs nutritives au moyen de la digestibilité in vitro en présence de jus de rumen. Les blocs à base de feuilles séchées de Moringa oleifera ou de tourteaux de coton et dont le liant est "goman" sont de meilleures caractéristiques physiques et nutritionnelles. Il n'y a pas de différence significative au niveau des caractéristiques physiques et nutritionnelles des BMN à base de tourteaux de coton et ceux à base de feuilles de Moringa oleifera.

(C) 2016 International Formulae Group. All rights reserved.

Mots clés : Blocs multi nutritionnels, liant, composition chimique, digestibilité in vitro, valeurs nutritives.

\section{Physical and nutritional characteristics of multi-nutritional blocks from agricultural and agro-industrial by-products in Benin}

\begin{abstract}
The agricultural and food sector generates numerous byproducts. These offer opportunities for choice of food supplements for ruminants during the dry season when grasses and other herbaceous forage are scarce
\end{abstract}


in rangelands. But, taken separately, these by-products have nutritional deficiencies or physical characteristics that contradict their use. The multi-nutritional blocks (MNB), are a means to address these constraints. Several formulations were tested using three types of binders (middlings of cassava called "garigo" cassava starch "goman" and clay). The hardness was estimated by exerting the thumb in the middle of the block after drying and cohesion under test to break by hand with greater effort. The color was appreciated by direct observation of the blocks and the smell through the smell. The chemical composition was determined according to official methods of $\mathrm{AOAC}$ and nutritive values using in vitro gas production. The BMN made from Moringa oleifera leaves or cotton cakes have presented the best physical and nutritional characteristics. It was also noticed that there was no significant difference in the physical and nutritional characteristics of BMN based on cotton cakes or the leaves of Moringa oleifera.

(C) 2016 International Formulae Group. All rights reserved.

Keywords: Multi-nutritional blocks, binder, chemical composition, in vitro digestibility, Benin.

\section{INTRODUCTION}

\begin{tabular}{llcr}
\multicolumn{2}{c}{ Dans de nombreux pays en } \\
développement, une bonne partie de \\
l'alimentation des ruminants est \\
principalement basée sur l'utilisation
\end{tabular} excessive des pâturages naturels et des résidus de culture (paille des céréales, fanes de légumineuses, etc.). Le principal problème de l'utilisation de ces fourrages réside dans leur déséquilibre nutritionnel, une faible teneur en protéines. Au Bénin, même pendant la saison des pluies, Babatoundé et al. (2011b) ont montré que les valeurs des matières azotées digestibles (MAD) dans les jachères pâturées par les taurillons Borgou n'excèdent pas 61 $\mathrm{g} / \mathrm{k}$ MO. Ces fourrages présentent également une faible digestibilité et un déficit en minéraux et en vitamines. Ces caractéristiques limitent les quantités ingérées de ces fourrages et permettent rarement d'atteindre un niveau alimentaire d'entretien (Moudjahed et al., 2000). Classiquement, l'amélioration de la valeur alimentaire de ces fourrages est obtenue par un apport d'énergie, de protéines pour activer la fermentation dans le rumen. Cependant, en Afrique subsaharienne, l'accès à ces compléments protéiques est plus difficile. Il se fait par l'achat d'aliments concentrés du commerce pour couvrir les besoins nutritifs des animaux sur pâturages ou pour l'engraissement à l'étable. Donc l'amélioration de la situation alimentaire du cheptel national nécessite le développement de système fourrager en adéquation avec les potentialités de l'élevage existant dans chaque zone agro écologique (Tabaï, 2009).
Pour maintenir les performances des animaux notamment en saison sèche, et satisfaire la demande sans cesse croissante en protéines animales, il s'avère nécessaire de trouver des pratiques alimentaires qui permettront d'éviter la pénurie alimentaire.

En Afrique de l'Ouest, le secteur agricole et agro-alimentaire génèrent de nombreux sousproduits (FAO, 2014). Ceux utilisés en élevage au Bénin ont été évalués à 11043 tonnes en 2010 (DE, 2012). Considérés séparément, ces sous-produits présentent des déficiences nutritionnelles ou des caractéristiques physiques qui contrarient leur utilisation. Les blocs alimentaires, communément appelés blocs multinutritionnels (BMN), restent un moyen pour corriger ces contraintes. Leur valorisation optimale pourrait à la fois améliorer les performances zootechniques des animaux et réduire les coûts alimentaires (Meffeja et al., 2007). La technique des blocs multi nutritionnels décrite par Sansoucy et al. (1986) et vulgarisée depuis lors par de nombreux organismes constitue un moyen de valorisation de sous-produits agricoles et agro-industriels. L'effet bénéfique de ces blocs multi nutritionnels a été signalé par Gnanda et al. (2015). Un premier objectif de cette étude est de fabriquer différents blocs alimentaires multi nutritionnels à partir des sous-produits agricoles et agro-industriels localement disponibles au Bénin. Le deuxième objectif est d'évaluer leurs caractéristiques physiques et nutritionnelles afin de les sélectionner et les insérer 
avantageusement dans les plans d'alimentation des ruminants domestiques.

\section{MATERIEL ET METHODES}

\section{Ingrédients alimentaires et proportions} incorporées dans les formules des blocs

Les fourrages (tiges de maïs, paille de riz, fanes d'arachide et niébé, les feuilles séchées de Moringa oleifera) et les sousproduits de transformations agroalimentaires tels que les sons (maïs, riz, sorgho, blé), les drêches (soja, niébé) ainsi que les tourteaux (coton, soja) sont les ingrédients utilisés pour la fabrication des blocs alimentaires multi nutritionnels (BMN). Le sel de cuisine, les coquilles d'huître ont été les minéraux additionnés aux ingrédients pré cités.

Sur la base de ces ingrédients, dix formules de BMN ont été proposées (Tableau 1). Les taux des ingrédients très fibreux (les tiges de maïs et la paille de riz) ont varié de 30 à $40 \%$ contre 15 à $20 \%$ pour les fanes séchées de légumineuses (arachide, niébé). Les feuilles séchées de Moringa oleifera, ingrédient important dans la formulation de ces blocs, ont été incorporées à des taux qui oscillent entre 10 et $15 \%$. A cause de la présence de $7,4 \mathrm{~g} / \mathrm{kg}$ de tannins dans les feuilles fraîches de Moringaoleifera (Okorondu et al., 2013), ces feuilles ont subi un séchage à l'ombre avant leur incorporation dans les formules des BMN. Le son de maïs a été uniquement utilisé dans la formule du BMN 8 (15\%), tandis que le tourteau de coton a représenté $10 \%(\mathrm{BMN} 4)$ et $15 \%(\mathrm{BMN} 10)$. Les drêches de soja, ayant présenté des niveaux élevés en MAT (près de $30 \%$ de MS) sont présentes dans toutes les formules (10 à $20 \%$ de taux d'incorporation). Les liants utilisés dans les différentes formulations ont été l'amidon de manioc appelé "goman", remoulage du manioc "garigo" et l'argile dans une proportion constante (10\%) avec une substitution de l'un ou l'autre selon les rations. Les coquilles d'huîtres et le sel de cuisine, également présents en proportion constante $(5 \%)$, ont été les ingrédients minéraux utilisés.

\section{Evaluation des caractéristiques physiques}

La dureté a été estimée en exerçant le pouce au milieu du bloc après séchage. Elle est bonne si le doigt ne s'enfonce pas ou avec une forte pression. Quant à la cohésion, elle a été déterminée en essayant de rompre le bloc à la main. Elle est bonne si le bloc ne se rompt pas ou avec un effort plus grand (Chehma et Senoussi, 2010). Pour la détermination du poids des BMN, dix (10) échantillons représentatifs de chaque type de bloc ont été pesés à l'aide d'une balance électronique de sensibilité $5 \mathrm{~g}$. Quant à la couleur, elle a été appréciée par observation directe des BMN et l'odeur, grâce à l'odorat.

\section{Détermination de la composition chimique et des valeurs nutritives}

Les échantillons de blocs fabriqués ont été soumis aux analyses chimiques de laboratoire. La matière sèche ( $\mathrm{MS}$, méthode ID 934,01), la matière organique (MO, méthode ID 942,05) et les matières azotées totales (MAT, méthode ID 954,01) ont été déterminées selon les méthodes officielles approuvées par AOAC (2000). Les teneurs en constituants pariétaux en particulier la cellulose brute (CB) a été déterminée selon la technique des sachets filtrants établie par ANKOM (ANKOM 200 Analyzer Fibre ; SKU: A200 et nb sp A200I). Par la suite, ces échantillons ont été soumis aux études de fermentescibilité in vitro en présence de jus de rumen de mouton et du tampon de Menke et al. (1979). Les échantillons de sous-produits (250 mg de MS) ont été incubés en seringues. L'incubation a été réalisée dans des seringues en verre de $100 \mathrm{ml}$ graduées par pas de $1 \mathrm{ml}$. Chaque seringue était équipée d'un robinet verrouillable à trois (3) voies. Les pistons ont été lubrifiés à la vaseline avant chaque incubation. Les mesures de fermentescibilité en seringues ont été toutes répétées au cours d'une seconde période et a permis de prendre en compte les déviations associées à des facteurs non contrôlables, tels que la variabilité du jus de rumen et les facteurs environnementaux. A chaque période d'essai, trois (3) répétitions d'un même échantillon de blocs ont été systématiquement effectuées. La 
position du piston était lue aux heures $0,2,4$, $6,8,12,24,48,72,96,120$, et 144 . Les volumes de gaz moyens produits ont été ajustés au modèle mathématique de France et al. (1993) :

$\mathrm{Y}=\mathrm{A}[1-\mathrm{e}[-\mathrm{b}(\mathrm{t}-\mathrm{T})-\mathrm{c}(\sqrt{ } \mathrm{t}-\sqrt{ } \mathrm{T})]]$ si $\mathrm{t} \geq \mathrm{T}$

$\mathrm{Y}=0$ si $0<\mathrm{t}<\mathrm{T}$

Y est le volume cumulé de gaz au temps $\mathrm{t}(\mathrm{ml})$, $\mathrm{t}$ le temps d'incubation (h), T est le temps de latence $(\mathrm{h}), \mathrm{A}(\mathrm{ml} / \mathrm{g}$ MS) est la production potentielle (maximale) de gaz c'est-à-dire l'asymptote ( $a u$ temps $t=\infty$ ), et $b$ (h-1) et c (h-1/2) sont des constantes.

L'équation du modèle établi une courbe cinétique théorique à partir des valeurs observées de volume de gaz. Le calcul a été réalisé par itérations successives jusqu'à la minimisation de la somme des carrés des écarts résiduels. Les paramètres issus de la modélisation ont été, le temps de latence, les volumes théoriques et les taux fractionnels de production de gaz.

En utilisant les équations de régression de Menke et Steingass (1988), le volume de gaz mesuré après 24 heures $\left(\mathrm{V}_{24 \mathrm{~h}}, \mathrm{ml} / 200 \mathrm{mg}\right.$ de MS) est associé aux MAT et $\mathrm{CT}(\mathrm{g} / \mathrm{kg} \mathrm{MS})$ pour estimer la digestibilité in vivo de la matière organique $(\mathrm{dMO})$ à partir de l'équation :

$\mathrm{dMO}(\%)=15,38+0,8453 \mathrm{~V} 24 \mathrm{~h}+0,0595$

$\mathrm{MAT}+0,0675 \mathrm{CT}$

$(\mathrm{R} 2=0,91 ; \mathrm{ETR}=4,48 ; \mathrm{n}=185)$
L'énergie métabolisable (EM), de même a été calculée selon Menke et Steingass (1988) à partir de la relation suivante :

$$
\begin{gathered}
\mathrm{EM}(\mathrm{kcal} / \mathrm{kg} \mathrm{MS})=(0,15+0,1557 \mathrm{dMO}+ \\
0,0130 \mathrm{CT}) \times 1000 / 4,18
\end{gathered}
$$

$\left(\mathrm{R}^{2}=0,95 ;\right.$ ETR $\left.=4,08 \mathrm{n}=185\right)$

Les valeurs énergétiques nettes ont été estimées dans le système français (INRA, 1988) des Unités Fourragères pour la Lactation (UFL) et pour l'entretien et l'engraissement (UFV). Les valeurs azotées ont été estimées selon l'équation de prédiction proposée par Jarrige et Demarquilly (1981) :

MAT $(\mathrm{g} / \mathrm{kg} \mathrm{MS})=0,929 \mathrm{MAT}-3,52$

\section{Analyses statistiques}

La statistique descriptive en termes de moyenne, d'écart-type et de coefficient de variation a été utilisée sur les données de composition chimique, des paramètres cinétiques de production de gaz et les valeurs nutritives des sous-produits. Le logiciel SAS (Statistical Analysis System) version 9,2 a été utilisé pour les analyses statistiques qui ont porté essentiellement sur des analyses de variance à un facteur (blocs). Les probabilités de signification associées aux valeurs $F$ de Fisher sont calculées. Les valeurs moyennes ont été comparées entre elles à l'aide du test de Student Newman Keuls au seuil de 5\% (Dagnelie, 1998).

Tableau 1 : Types de formules avec les proportions des différents ingrédients ( $\%$ de MF).

\begin{tabular}{lcccccccccc}
\hline Ingrédients & $\mathbf{1}$ & $\mathbf{2}$ & $\mathbf{3}$ & $\mathbf{4}$ & $\mathbf{5}$ & $\mathbf{6}$ & $\mathbf{7}$ & $\mathbf{8}$ & $\mathbf{9}$ & $\mathbf{1 0}$ \\
\hline Tige de maïs & 40 & 40 & 40 & 0 & 0 & 0 & 0 & 30 & 30 & 30 \\
Paille de riz & 0 & 0 & 0 & 40 & 40 & 40 & 30 & 0 & 0 & 0 \\
Fanes de niébé & 20 & 20 & 20 & 0 & 0 & 0 & 0 & 0 & 0 & 0 \\
Fanes d'arachide & 0 & 0 & 0 & 20 & 20 & 20 & 20 & 0 & 15 & 15 \\
Feuilles de Moringa & 10 & 10 & 10 & 0 & 10 & 10 & 15 & 15 & 10 & 0 \\
Son de maïs & 0 & 0 & 0 & 0 & 0 & 0 & 0 & 15 & 0 & 0 \\
Tourteau de coton & 0 & 0 & 0 & 10 & 0 & 0 & 0 & 0 & 0 & 15 \\
Drêches de soja & 10 & 10 & 10 & 10 & 10 & 10 & 15 & 20 & 20 & 20 \\
Goman* & 10 & 0 & 0 & 10 & 10 & 10 & 0 & 10 & 10 & 10 \\
Garigo** & 0 & 10 & 0 & 0 & 0 & 0 & 0 & 0 & 0 & 0 \\
Argile & 0 & 0 & 10 & 0 & 0 & 0 & 10 & 0 & 0 & 0 \\
Coquilles d'huîtres & 5 & 5 & 5 & 5 & 5 & 5 & 5 & 5 & 5 & 5 \\
Sel de cuisine & 5 & 5 & 5 & 5 & 5 & 5 & 5 & 5 & 5 & 5 \\
\hline
\end{tabular}

*Goman : Amidon de manioc en langue locale Fon ; ** Garigo : remoulage farine de manioc en langue locale Fon. 


\section{RESULTATS \\ Caractéristiques physiques des blocs alimentaires multi nutritionnels}

Les caractéristiques physiques des blocs fabriqués sont présentées dans le Tableau 2. Les poids ont peu varié (633 g en moyenne). Les dimensions des blocs ont été constantes en raison du fait que c'est le même moule qui a été utilisée pour toutes les préparations et la technique de moulage utilisée a été constante (3 remplissages et 3 pressions exercées avec les pressoirs).

Les blocs à base de tige de maïs et d'amidon de manioc "goman" (BMN1, BMN8, BMN9 et BMN10) ont présenté une meilleure dureté et cohésion. Ceux à base de paille de riz ont été moins durs avec une faible cohésion. Avec le remoulage du manioc "garigo" la dureté et la cohésion des $\mathrm{BMN}$ sont moyennes. Ceux à base de paille de riz et d'argile (taux d'incorporation de $10 \%$ dans le mélange) sont moins durs et s'effritent au bout de quelques jours. La couleur des BMN a varié du claire au foncée en passant par le clair foncé.

\section{Composition chimique}

Les teneurs en matière sèche (MS), en cendres totales (CT) et en matière organique (MO) des BMN fabriqués ne sont pas significativement différentes $(\mathrm{P}>0,05)$ (Tableau 3). Par contre, les constituants de la matière organique en particulier la cellulose brute (CB) et les matières azotées totales (MAT) ont très significativement variés selon le type de blocs $(\mathrm{P}<0,001)$. Avec une valeur de $\mathrm{F}=243,63$, les MAT sont les constituants les plus influencés par le type de BMN fabriqué. Les teneurs en MAT étaient en moyenne plus élevées dans les BMN8 et BMN10 (14,7 et 14,5\% MS respectivement), tandis que les BMN2 et BMN6 ont présenté les taux les plus faibles $(9,3 \%$ de $\mathrm{MS})$. L'incorporation des taux plus élevés en feuilles de Moringa oleifera (15\%) et en drêches de soja $(20 \%)$ améliore de 5 points les teneurs en MAT de ces BMN. En général, les BMN fabriqués sont riches en fibres avec des teneurs en CB supérieures à 20\% de MS.

\section{Caractéristiques de fermentescibilité in vitro en présence du jus de rumen}

Le temps de latence c'est-à-dire le délai nécessaire à la population microbienne adhérente aux constituants pariétaux des BMN pour se développer et augmenter sa concentration en enzymes a été très faible (moins d'une heure) notamment pour les BMN4, BMN8, BMN9 et BMN10 (Tableau 4). Le processus fermentaire s'est donc déroulé très rapidement comme l'ont montré les taux fractionnels de production de gaz au temps $T / 2(\mu \mathrm{T} / 2)$ pour lequel on obtient la moitié de la production maximale de gaz. Les volumes finaux de gaz produits ont été élevés (plus de $200 \mathrm{ml} / \mathrm{g}$ de MS) sauf pour les BMN3 et BMN9 qui ont présenté des volumes plus faibles. Quant à la dégradabilité théorique (DT), elle a été en moyenne de $40,86 \%$. Quel que soit le type de BMN, la production de gaz qui est régulière et croissante les trois (3) premiers jours d'incubation a été considérablement ralentie au-delà de cette période. Ainsi, après 72 heures d'incubation, la phase asymptotique a été presque atteinte et l'essentiel du volume total de gaz a été produit (Figure 1). Les blocs multi nutritionnels de type BMN8 sont beaucoup plus fermentescibles que les autres BMN.

\section{Valeurs nutritives}

Les valeurs nutritives des blocs multi nutritionnels fabriqués sont présentées au Tableau 5. La digestibilité de la matière organique (dMO) des BMN a varié de 55,1 à $70,4 \%$. En moyenne, les BMN dosent $60 \%$ de dMO. Aux valeurs de dMO élevées correspondent également des valeurs UFL et UFV élevées. Tous les modèles de BMN fabriqués sont bien pourvus en énergies nettes $(0,80$ UFL et $0,72 \mathrm{UFV} / \mathrm{kg}$ MS en moyenne). Le BMN4 a été le plus digestible et le plus énergétique. En ce qui concerne les teneurs en matières azotées digestibles (MAD/UFL), les BMN ont présenté des valeurs généralement inférieures à $100 \mathrm{~g} / \mathrm{kg}$ de MS. Il est intéressant de constater que les BMN 7, 8, 9 et 10 ont présenté des valeurs supérieures à cette limite. Un rapport MAD/UFL de $126,6 \mathrm{~g} / \mathrm{kg}$ de MS a même été obtenu pour le BMN 10. 
Tableau 2 : Caractéristiques physiques des blocs.

\begin{tabular}{lllllll}
\hline BMN & Poids $(\mathbf{g})$ & $\begin{array}{c}\text { Dimensions } \\
(\mathbf{L x l} \mathbf{n})(\mathbf{C m})\end{array}$ & Dureté & Cohésion & Couleur & Odeur \\
\hline 1 & 592,26 & $16 \times 16 \times 13$ & $* * *$ & $* * *$ & Claire foncée & Bonne \\
2 & 611,08 & $16 \times 16 \times 13$ & $* *$ & $*$ & Claire foncée & Bonne \\
3 & 693,58 & $16 \times 16 \times 13$ & $* *$ & $*$ & Claire foncée & Bonne \\
4 & 619,65 & $16 \times 16 \times 13$ & $* *$ & $* *$ & Foncée & Bonne \\
5 & 642,50 & $16 \times 16 \times 13$ & $*$ & $*$ & Claire foncée & Bonne \\
6 & 625,42 & $16 \times 16 \times 13$ & $*$ & $*$ & Claire foncée & Bonne \\
7 & 555,00 & $16 \times 16 \times 13$ & $*$ & $*$ & Claire foncée & Bonne \\
8 & 645,80 & $16 \times 16 \times 13$ & $* *$ & $* * *$ & Claire foncée & Bonne \\
9 & 726,70 & $16 \times 16 \times 13$ & $* *$ & $* * *$ & Claire foncée & Bonne \\
10 & 615,00 & $16 \times 16 \times 13$ & $* *$ & $* * *$ & Foncée & Bonne \\
\hline
\end{tabular}

BMN : Blocs alimentaires Multi Nutritionnels. Dureté : Bonne (***); Moyenne (**); Médiocre $\left.{ }^{*}\right)$.

Cohésion : Bonne $(* * *)$; Moyenne $(* *)$; Médiocre $(*)$. Cm : Centimètre.

Tableau 3 : Composition chimique des blocs/BMN (\% de MS).

\begin{tabular}{llllll}
\hline \multicolumn{1}{c}{ BMN } & \multicolumn{1}{c}{ MS } & \multicolumn{1}{c}{ CT } & \multicolumn{1}{c}{ MO } & MAT & CB \\
\hline BMN 1 & $96,40 \pm 0,09^{\mathrm{a}}$ & $17,99 \pm 1,07^{\mathrm{a}}$ & $82,01 \pm 1,07^{\mathrm{a}}$ & $10,47 \pm 0,03^{\mathrm{e}}$ & $22,47 \pm 0,00^{\mathrm{a}}$ \\
BMN 2 & $87,12 \pm 0,03^{\mathrm{a}}$ & $18,40 \pm 0,71^{\mathrm{a}}$ & $81,60 \pm 0,71^{\mathrm{a}}$ & $9,32 \pm 0,23^{\mathrm{e}}$ & $21,81 \pm 0,08^{\mathrm{a}}$ \\
BMN 3 & $92,39 \pm 3,82^{\mathrm{a}}$ & $27,31 \pm 0,89^{\mathrm{a}}$ & $72,69 \pm 0,89^{\mathrm{a}}$ & $10,30 \pm 0,05^{\mathrm{d}}$ & $21,42 \pm 0,21^{\mathrm{b}}$ \\
BMN 4 & $94,65 \pm 9,62^{\mathrm{a}}$ & $37,66 \pm 1,59^{\mathrm{a}}$ & $62,34 \pm 1,59^{\mathrm{a}}$ & $10,98 \pm 0,21^{\mathrm{c}}$ & $14,72 \pm 0,01^{\mathrm{f}}$ \\
BMN 5 & $92,16 \pm 0,55^{\mathrm{a}}$ & $22,64 \pm 0,36^{\mathrm{a}}$ & $77,36 \pm 0,36^{\mathrm{a}}$ & $7,40 \pm 0,36^{\mathrm{f}}$ & $22,37 \pm 0,22^{\mathrm{a}}$ \\
BMN 6 & $93,81 \pm 0,33^{\mathrm{a}}$ & $21,89 \pm 0,59^{\mathrm{a}}$ & $78,11 \pm 0,59^{\mathrm{a}}$ & $9,37 \pm 0,08^{\mathrm{e}}$ & $22,38 \pm 0,46^{\mathrm{a}}$ \\
BMN 7 & $92,67 \pm 0,27^{\mathrm{a}}$ & $30,46 \pm 0,50^{\mathrm{a}}$ & $69,54 \pm 0,50^{\mathrm{a}}$ & $13,65 \pm 0,01^{\mathrm{b}}$ & $18,21 \pm 0,00^{\mathrm{d}}$ \\
BMN 8 & $95,00 \pm 0,26^{\mathrm{a}}$ & $19,13 \pm 0,10^{\mathrm{a}}$ & $80,87 \pm 0,10^{\mathrm{a}}$ & $14,71 \pm 0,17^{\mathrm{a}}$ & $17,04 \pm 0,00^{\mathrm{e}}$ \\
BMN 9 & $95,19 \pm 0,71^{\mathrm{a}}$ & $21,94 \pm 0,58^{\mathrm{a}}$ & $78,06 \pm 0,58^{\mathrm{a}}$ & $13,65 \pm 0,05^{\mathrm{b}}$ & $20,04 \pm 0,00^{\mathrm{c}}$ \\
BMN 10 & $94,86 \pm 0,00^{\mathrm{a}}$ & $18,32 \pm 0,46^{\mathrm{a}}$ & $81,68 \pm 0,46^{\mathrm{a}}$ & $14,46 \pm 0,07^{\mathrm{a}}$ & $20,29 \pm 0,00^{\mathrm{c}}$ \\
\hline Moyenne & $\mathbf{9 3 , 4 2}$ & $\mathbf{2 3 , 5 7}$ & $\mathbf{7 6 , 4 3}$ & $\mathbf{1 1 , 4 3}$ & $\mathbf{2 0 , 0 8}$ \\
F & $0,93^{\mathrm{NS}}$ & $1,32^{\mathrm{NS}}$ & $1,32^{\mathrm{NS}}$ & $243.63^{*} * *$ & $218.61^{* * *}$ \\
CV (\%) & 2,0 & 21,0 & 6,5 & 18,8 & 10,3 \\
\hline
\end{tabular}

Les valeurs suivies de la même lettre dans la même colonne ne sont pas significativement différentes selon le test de Student Newman-Keuls $(\mathrm{P}<0,05) \mathrm{NS}$ : non significatif au seuil de $5 \%$; $: \mathrm{P}<0,05 ; * *: \mathrm{P}<0,01 ; * * *: \mathrm{P}<0,001$. CV : Coefficient de variation, MS : Matière Sèche, MO : Matière Organique, MAT : Matières Azotées Totales, CB : Cellulose Brute. 
Tableau 4 : Paramètres cinétiques de production de gaz.

\begin{tabular}{llllll}
\hline BMN & \multicolumn{1}{c}{ Latence (h) } & \multicolumn{1}{c}{ Temps T/2 (h) } & \multicolumn{1}{c}{$\boldsymbol{\mu}_{\mathbf{T} / \mathbf{2}}(\mathbf{\%} / \mathbf{h})$} & $\mathbf{V}_{\text {final }}(\mathbf{m l} / \mathbf{g ~ M S})$ & $\mathbf{D T}(\mathbf{\%} \mathbf{M S})$ \\
\hline BMN 1 & $1,18 \pm 0,05^{\mathrm{ab}}$ & $20,92 \pm 2,38^{\mathrm{ab}}$ & $0,027 \pm 0,00^{\mathrm{ab}}$ & $239,29 \pm 17,35^{\mathrm{abc}}$ & $43,21 \pm 1,34^{\mathrm{ab}}$ \\
BMN 2 & $1,14 \pm 0,05^{\mathrm{ab}}$ & $22,84 \pm 1,42^{\mathrm{a}}$ & $0,022 \pm 0,00^{\mathrm{b}}$ & $259,76 \pm 14,9^{\mathrm{ab}}$ & $43,95 \pm 1,78^{\mathrm{a}}$ \\
BMN 3 & $1,99 \pm 0,24^{\mathrm{a}}$ & $20,56 \pm 2,17^{\mathrm{ab}}$ & $0,033 \pm 0,00^{\mathrm{ab}}$ & $196,56 \pm 11,08^{\mathrm{cd}}$ & $42,52 \pm 0,55^{\mathrm{ab}}$ \\
BMN 4 & $0,44 \pm 0,21^{\mathrm{b}}$ & $14,04 \pm 2,11^{\mathrm{bc}}$ & $0,038 \pm 0,00^{\mathrm{ab}}$ & $205,94 \pm 20,59^{\mathrm{bcd}}$ & $34,81 \pm 1,11^{\mathrm{c}}$ \\
BMN 5 & $0,92 \pm 0,09^{\mathrm{b}}$ & $17,36 \pm 1,45^{\mathrm{ab}}$ & $0,028 \pm 0,00^{\mathrm{ab}}$ & $203,65 \pm 4,49^{\mathrm{bcd}}$ & $42,90 \pm 0,78^{\mathrm{ab}}$ \\
BMN 6 & $0,87 \pm 0,29^{\mathrm{b}}$ & $20,87 \pm 2,11^{\mathrm{ab}}$ & $0,023 \pm 0,00^{\mathrm{b}}$ & $200,15 \pm 11,25^{\mathrm{bcd}}$ & $42,10 \pm 0,78^{\mathrm{ab}}$ \\
BMN 7 & $1,28 \pm 0,08^{\mathrm{ab}}$ & $20,57 \pm 0,79^{\mathrm{ab}}$ & $0,026 \pm 0,00^{\mathrm{b}}$ & $211,14 \pm 11,32^{\mathrm{bcd}}$ & $40,54 \pm 0,76^{\mathrm{abc}}$ \\
BMN 8 & $0,48 \pm 0,10^{\mathrm{b}}$ & $13,49 \pm 0,45^{\mathrm{bc}}$ & $0,035 \pm 0,00^{\mathrm{ab}}$ & $270,22 \pm 6,86^{\mathrm{a}}$ & $40,56 \pm 3,71^{\mathrm{abc}}$ \\
BMN 9 & $0,62 \pm 0,61^{\mathrm{b}}$ & $9,88 \pm 1,23^{\mathrm{bc}}$ & $0,052 \pm 0,01^{\mathrm{ab}}$ & $172,80 \pm 4,10^{\mathrm{d}}$ & $35,85 \pm 1,77^{\mathrm{bc}}$ \\
BMN 10 & $0,64 \pm 0,09^{\mathrm{b}}$ & $15,01 \pm 0,40^{\mathrm{c}}$ & $0,042 \pm 0,00^{\mathrm{a}}$ & $211,16 \pm 18,3^{\mathrm{bcd}}$ & $42,20 \pm 0,84^{\mathrm{ab}}$ \\
\hline Moyenne & $\mathbf{0 , 9 6}$ & $\mathbf{1 7 , 5 5}$ & $\mathbf{0 , 0 3}$ & $\mathbf{2 1 7 , 0 7}$ & $\mathbf{4 0 , 8 6}$ \\
F & $3,67^{*}$ & $6,96^{* * *}$ & $3,06^{*}$ & $5,21 * * *$ & $3,74 * *$ \\
CV (\%) & 44,4 & 15,9 & 28,4 & 10,5 & 6,8 \\
\hline
\end{tabular}

Les valeurs suivies de la même lettre dans la même colonne ne sont pas significativement différentes selon le test de Student Newman-Keuls $(\mathrm{P}<0,05)$.ns : non significatif au seuil de $5 \%$; $: \mathrm{P}<0,05 ; * *: \mathrm{P}<0,01 ; * * *: \mathrm{P}<0,001$.

Tableau 5 : Digestibilité et valeur nutritive des blocs /BMN.

\begin{tabular}{|c|c|c|c|c|c|}
\hline \multirow{3}{*}{$\mathbf{B M N}$} & \multirow{3}{*}{ dMO (\% MS) } & \multicolumn{4}{|c|}{ Valeur nutritive } \\
\hline & & \multicolumn{2}{|c|}{ Valeur énergétique } & \multicolumn{2}{|c|}{ Valeur azotée } \\
\hline & & UFL & UFV & MAD (g/kg MS) & MAD/UFL \\
\hline BMN 1 & $55,11 \pm 0,31^{\mathrm{c}}$ & $0,72 \pm 0,00^{c}$ & $0,62 \pm 0,00^{c}$ & $50,01 \pm 0,29^{\mathrm{e}}$ & $69,92 \pm 0,41^{\mathrm{d}}$ \\
\hline BMN 2 & $55,89 \pm 0,80^{\mathrm{c}}$ & $0,73 \pm 0,01^{\mathrm{c}}$ & $0,64 \pm 0,01^{\mathrm{c}}$ & $51,40 \pm 2,14^{\mathrm{e}}$ & $70,67 \pm 2,94^{\mathrm{d}}$ \\
\hline BMN 3 & $62,36 \pm 4,53^{b}$ & $0,84 \pm 0,07^{\mathrm{b}}$ & $0,77 \pm 0,08^{\mathrm{b}}$ & $60,47 \pm 0,48^{\mathrm{d}}$ & $72,05 \pm 0,57^{\mathrm{d}}$ \\
\hline BMN 4 & $70,40 \pm 0,18^{\mathrm{a}}$ & $0,98 \pm 0,00^{\mathrm{a}}$ & $0,94 \pm 0,00^{\mathrm{a}}$ & $66,81 \pm 2,00^{\mathrm{c}}$ & $68,22 \pm 2,04^{\mathrm{d}}$ \\
\hline BMN 5 & $55,07 \pm 0,39^{\mathrm{c}}$ & $0,72 \pm 0,01^{\mathrm{c}}$ & $0,63 \pm 0,01^{\mathrm{c}}$ & $33,55 \pm 3,34^{\mathrm{f}}$ & $46,30 \pm 4,62^{\mathrm{f}}$ \\
\hline BMN 6 & $54,0 \pm 01,67^{\mathrm{c}}$ & $0,70 \pm 0,02^{\mathrm{c}}$ & $0,61 \pm 0,03^{\mathrm{c}}$ & $51,88 \pm 0,78^{\mathrm{e}}$ & $73,71 \pm 1,10^{\mathrm{d}}$ \\
\hline BMN 7 & $63,56 \pm 0,76^{\mathrm{b}}$ & $0,86 \pm 0,00^{\mathrm{b}}$ & $0,79 \pm 0,01^{\mathrm{b}}$ & $91,62 \pm 0,13^{\mathrm{b}}$ & $106,50 \pm 0,15^{\mathrm{c}}$ \\
\hline BMN 8 & $65,80 \pm 0,34^{\mathrm{b}}$ & $0,88 \pm 0,01^{\mathrm{b}}$ & $0,82 \pm 0,01^{\mathrm{b}}$ & $101,44 \pm 1,62^{\mathrm{a}}$ & $115,18 \pm 1,84^{\mathrm{b}}$ \\
\hline BMN 9 & $60,06 \pm 1,08^{b c}$ & $0,80 \pm 0,02^{b c}$ & $0,72 \pm 0,02^{b c}$ & $91,63 \pm 0,45^{\mathrm{b}}$ & $115,25 \pm 0,56^{\mathrm{b}}$ \\
\hline BMN 10 & $59,58 \pm 0,80^{\mathrm{bc}}$ & $0,78 \pm 0,00^{\mathrm{bc}}$ & $0,71 \pm 0,00^{\mathrm{bc}}$ & $99,12 \pm 0,70^{\mathrm{a}}$ & $126,58 \pm 0,90^{\mathrm{a}}$ \\
\hline Moyenne & 60,18 & $\mathbf{0 , 8 0}$ & 0,72 & 69,79 & 86,44 \\
\hline $\mathrm{F}$ & $11,09 * * *$ & $12,61 * * *$ & $12,50 * * *$ & $243.67 * * *$ & $178.83 * * *$ \\
\hline CV (\%) & 4,6 & 5,4 & 7,1 & 30,0 & 27,2 \\
\hline
\end{tabular}

Les valeurs suivies de la même lettre dans la même ligne ne sont pas significativement différentesselon le test de Student Newman-Keuls $(\mathrm{P}<0,05)$; * :P $<0,05 ; * *: \mathrm{P}<0,01 ; * * *: \mathrm{P}<0,001$. 


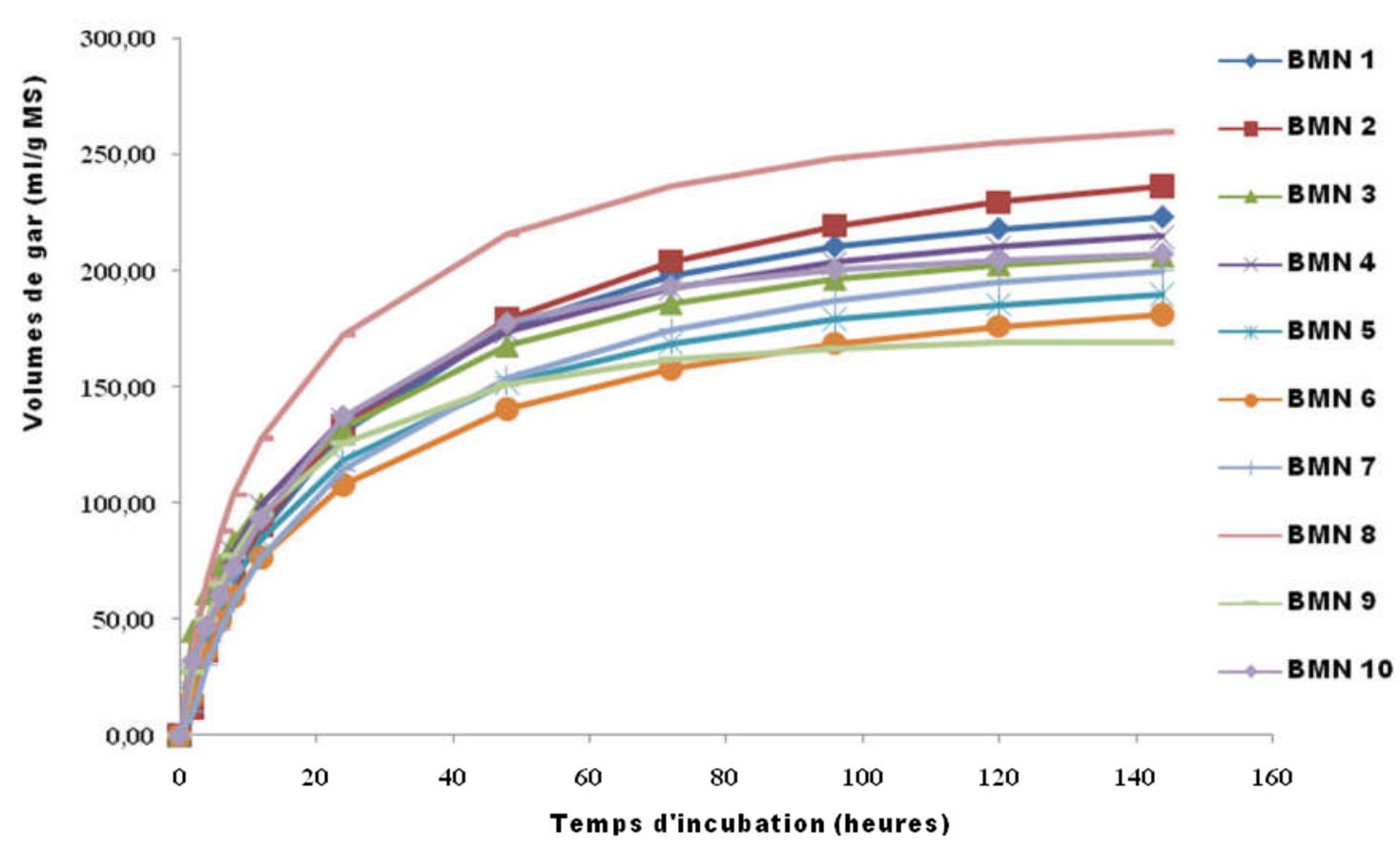

Figure 1 : Cinétiques de fermentescibilité en seringues des blocs.

\section{DISCUSSION}

\section{Caractéristiques physiques}

La fabrication des blocs BMN a été artisanale. Dans ce cas, Herrera et al. (2007) soulignent que les caractéristiques physiques des BMN constituent un aspect important à considérer. La dureté des BMN est un facteur qui détermine nettement leur consommation par l'animal (Mwendia et Khasataili, 1990 ; Hadjipanayiotou et al., 1993 ; Birbe et al., 1994). Herrera et al. (2007) ont observé que la consommation animale diminue au fur et à mesure que la dureté des BMN augmente. Pour cette étude, les niveaux d'incorporation de feuilles de Moringa oleifera ont varié de 0 à $15 \%$. Ces résultats nous allient avec ceux rapportés par Chehma et Senoussi (2010) sur les blocs avec inclusion des rebuts de dattes. La présence ou l'absence des feuilles de Moringa oleifera dans les préparations des BMN a beaucoup influencé la couleur des BMN fabriqués (claire pour les blocs sans Moringa oleifera et foncée pour les formules l'incluant). Quant à l'odeur, elle a été très bonne surtout pour les formules où le tourteau de coton a été substitué par les feuilles séchées de Moringa oleifera. Ces résultats sont semblables à ceux obtenus par Zogang et al. (2012) sur les BMN en incluant différents niveaux de feuilles de Tithonia diversifolia. En effet, cette modification de couleur de clair à foncée est liée à la présence des pigments chlorophylliens dans les feuilles de Moringa oleifera. La variation de la couleur se justifie par l'ajout de niveaux croissant de feuilles de Moringa oleifera dans la fabrication des BMN.

\section{Composition chimique et valeurs nutritives}

Avec les niveaux d'incorporation des ingrédients protéiniques constitués de fanes de légumineuses (niébé, arachide), des drêches de soja et de feuilles séchées de Moringa oleifera n'excédant pas les $20 \%$, les teneurs en MAT des BMN fabriqués ont varié de 9,32 à $14,71 \%$ de MS sans jamais excéder les $15 \%$. La même fourchette de teneur en MAT a été obtenue par Chehma et Senoussi (2010) 
suite à l'introduction des quantités variables de 25 à $75 \%$ de rebuts de dattes dans les BMN fabriqués. En utilisant les feuilles séchées de Sida cordifolia, une plante invasive du Niger, Soumana et al. (2016), n'ont pas pu obtenir également les teneurs élevées en azote dans les BMN fabriqués (7,46 à 11,60\% de MS). Avec $35 \%$ de feuilles de Moringaoleiferaincorporées dans la fabrication des BMN, des niveaux azotés élevés $(22 \%$ en MAT) ont été obtenus par Asaolu (2012). Rabéha et al. (2010) ont pu relever le niveau de MAT à $32 \%$ de MS en incorporant $65 \%$ de rebuts de dattes dans la fabrication des BMN. Avec 3,4\% de farine de viande et $25 \%$ de gros son, la même teneur de MAT $=32 \%$ a été obtenue par Houmani et Tisserand (1999). En réalité, le niveau azoté des BMN est lié à la nature d'ingrédient protéinique ainsi qu'à la dose incorporée. L'incorporation des taux plus élevés en feuilles de Moringa oleifera (15\%) améliore de 5 points les teneurs en MAT de ces BMN. Ces feuilles de Moringa oleifera viennent substituer les tourteaux de coton dans les formules de BMN. L'effet bénéfique de la substitution des feuilles séchées de légumineuses ligneuses par les tourteaux de coton dans l'alimentation des animaux domestiques a été déjà signalé par Defang et al. (2014).

Les BMN fabriqués sont plus riches en fibres avec des teneurs en cellulose brute (CB) oscillant autour de $20 \%$ de MS. Au fur et à mesure que les taux d'incorporation des aliments fibreux (tiges de maiis et fanes de légumineuses) augmentent, les teneurs en $\mathrm{CB}$ s'élèvent également variant de ce fait, de 14,7 à $22,5 \%$ de MS. Ces résultats nous allient à ceux rapportés récemment par Soumana et al. (2016) sur les BMN fabriqués à base de feuilles séchées de Sida cordifolia.

D'après plusieurs nutritionnistes (Demarquilly, 1981 ; INRA, 2007 ; Sauvant et al., 2004), il existe une relation étroite entre la digestibilité de la matière organique (dMO) des aliments et leurs teneurs en fibres. Les
BMN dont les teneurs en $\mathrm{CB}$ sont faibles ont présenté les dMO élevées. En général, quand le niveau de digestibilité de la matière organique est supérieur à $60 \%$, l'aliment est au-dessus du niveau entretien. Moins que cette valeur, les BMN fabriqués sont mauvais et les modèles BMN1, BMN2, BMN5 et BMN6 confirment cette tendance.

Les travaux de Babatoundé et al. (2011a) sur l'engraissement des ovins Djallonké à l'herbage indiquent qu'il faut 0,60 UF comme teneur en énergie nette et un rapport MAD/UF de l'ordre de $90 \mathrm{~g}$ par kg de MS pour obtenir des gains moyens quotidiens (GMQ) de $50 \mathrm{~g} / \mathrm{j}$ chez les moutons Djallonké. Avec les valeurs UFL et les rapports MAD/UFL présentés par les BMN7, BMN8, BMN9 et BMN10, on peut espérer des gains moyens quotidiens au-delà de cette limite. Selon Rivière (1991), il faut 0,80 UFL/kg MS et un rapport $\mathrm{MAD} / \mathrm{UFL}=120 \mathrm{~g}$ pour obtenir en milieu tropical des GMQ de l'ordre de 100 $\mathrm{g} / \mathrm{j}$ sur l'engraissement des ovins Djallonké. Il est intéressant de constater que les modèles BMN9 et BMN10 des BMN fabriqués permettent d'atteindre cette performance. Aussi bien au niveau de leurs digestibilités que de leurs teneurs en énergie nette (UFL) et en rapport $\mathrm{MAD} / \mathrm{UFL}$, les modèles de $\mathrm{BMN}$ fabriqués (BMN1, BMN2, BMN5 et BMN6) ne donnent pas satisfaction. Par conséquent, ils ne peuvent pas assurer une complémentation adéquate aux ruminants si l'objectif fixé est de les engraisser.

\section{Conclusion}

La présente étude a montré qu'il est techniquement possible de fabriquer des blocs multi nutritionnels à base des ressources alimentaires localement disponibles au Bénin. Plusieurs formules ont été testées, mais les blocs alimentaires multi nutritionnels BMN8 (à base de tiges et son de maïs, de Moringa oleifera et de drêches de soja) et le BMN10 (à base de tiges, de fanes d'arachide, de tourteau de coton et de drêches de soja) ont présentés les meilleures caractéristiques physiques et 
nutritionnelles.

Aucune

différence

significative n'a été notée au niveau des caractéristiques physiques et nutritionnelles des BMN à base de tourteaux de coton et des BMN à base de feuilles séchées de Moringa oleifera. En définitive, cette étude montre que les blocs multi nutritionnels constituent une alternative pratique et efficace pour complémenter les régimes alimentaires pauvres des ruminants, surtout au cours des périodes difficiles (saison sèche notamment).

\section{CONFLIT D'INTERETS}

Les auteurs déclarent qu'il n'y a pas de conflits d'intérêts entre eux.

\section{CONTRIBUTIONS DES AUTEURS}

MM a contribué à la rédaction du protocole expérimental qui entre dans le cadre de ses travaux de thèse de doctorat et à la rédaction de la première mouture de cet article. SB à opérer les moutons Djallonké et à poser les canules du rumen qui ont servi aux études de fermentescibilité in vitro en présence de jus de rumen. Il a aussi participé en tant qu'auteur correspondant à l'amendement de la partie composition chimique et valeurs nutritives des blocs, à l'analyse statistique ainsi qu'à l'interprétation des données de l'étude. ABA et MJDB ont contribué à la mise au point des formules de BMN et aux travaux de laboratoire aboutissant à la détermination de la composition chimique ainsi qu'aux études de fermentescibilité in vitro en présence du jus de rumen. CAAMC et GAM ont dirigé les travaux de recherche et ont contribué à la relecture du manuscrit.

\section{REMERCIEMENTS}

Les auteurs remercient tout le personnel du Laboratoire de Zootechnie de la Faculté des Sciences Agronomiques de l'Université d'Abomey-Calavi, les éleveurs et agro-éleveurs de la commune de Gogounou pour leur contribution à cette étude.

\section{RÉFÉRENCES}

AOAC. 2000. Official Methods of Analysis $\left(13^{\text {th }}\right.$ edn). Association of Official Analytical Chemist: Washington, DC.

Asaolu VO. 2012. Development of moringa multi-nutrient block as dry season feed supplement for ruminants. Livest. Res. Rur. Dev., 24: 46.

Babatounde B, Oumorou M, Alkoiret TI, Vidjannagni S, Mensah GA. 2011a. Relative Frequencies, Chemical Composition and in vitro Organic Matter Digestibility of Forage Consumed by Sheep in Humid Tropic of West Africa. Journal of Agricultural Science and Technology, A1: 39-47.

Babatounde S, Sidi H, Houinato M, Oumorou M, Mensah GA, Sinsin BA. 2011b. Valeur Alimentaire des fourrages consommés par les taurillons Borgou sur les parcours naturels du centre du Bénin. Int. J. Biol. Chem. Sci., 5(6): 2382-2394.

Birbe B, Chacon E, Taylhardat L, Garmendia J, Mata D. 1994. Aspectosfisicos de Importancia en la fabricacion $\mathrm{y}$ utilizacion de bloques multinutricionales. In I Conferencia Internacional Bloques Multinutricionales, Cardozo A, Birbe B (eds). Guanare, Venezuela; $1-14$.

Chehma A, Senoussi A. 2010. Fabrication de blocs multi nutritionnels (BMN) à base de Sous-produits de palmier dattier et d'urée. Livest. Res. Rur. Dev., 22(4).

Dagnelie P. 1998. Statistique Théorique et Appliquée : Inférences statistiques à une et deux dimensions (Tome 2). De Boeck et Larcier: Paris-Bruxelles.

DE (Direction de L’Elevage du Bénin). 2012. Annuaire statistique, Cotonou.

Defang HF, Keambou TC, Manjeli Y, Teguia A, Pamo TE. 2014. Influence de la farine des feuilles de Leucaena leucocephala sur les performances de croissance des lapereaux. Int. J. Biol. Chem. Sci., 8(4): 1430-1437.

Demarquilly C, Jarrige R. 1981. Prévision de la Valeur Nutritive des Aliments des Ruminants. Editions INRA de Paris: 4156. 
FAO. 2014. Résidus agricoles et sous-produits agroindustriels en Afrique de l'Ouest : État des lieux et perspectives pour l'élevage. Bureau Régional pour l'Afrique de la FAO, Accra.

France J, Dhanoa MS, Theodorou MK, Lister SJ, Davies DR, Isaac D. 1993. A model to interpret gas accumulation profiles associated with in vitro degradation of ruminant feeds. J. Theor. Biol., 163: 99 111.

Gnanda IB, Bougouma-Yaméogo VMC, N'diaye W, Ouedraogo T, Kaboré A, Lodoun B, Sinon B. 2015. L'embouche bovine dans les élevages du Plateau Central du Burkina Faso : Résultats économiques d'une démarche de validation d'un référentiel technicoéconomique sur la spéculation. Int. $J$. Biol. Chem. Sci., 9(6): 2648-2662.

Hadjipanayiotou M, Verhaeghe L, Kronfoleha R, Labban LM, Amin M, Al-Wadi M, Badran A, Dawa K, Shurbaji A, Houssein M, Malki G, Naigm T, Merawi AR, Harres AK. 1993b. Urea blocks II Performance of cattle and sheep offered urea blocks in Syria. Livest. Res. Rur. Dev., 5: 1-16.

Herrera P, Birbe B, Dominguez C, Martinez N. 2007. Experiences with multinutrient blocks in Venezuelan tropics. In Feed supplementation Blocks. Urea-molasses multinutrient blocks: simple and effective feed supplement technology for ruminant agriculture Makkar, HPS, Sanchez M, Speedy AW (eds). FAO, Animal Production and Health, 1-12.

Houmani M, Tisserand JL. 1999. Complémentation d'une paille de blé avec des blocs multinutritionnels : effets sur la digestibilité de la paille et intérêt pour des brebis taries et des agneaux en croissance. Annales de Zootechnie, 48(3): 199-209.

INRA. 1988. Alimentation des bovins, ovins et caprins. Ouvrage collective dirigé par Jarrige R (ed). IRNRA : Paris France.

INRA. 2007. Alimentation des Bovins, Ovins et Caprins: Besoins des Animaux-
Valeurs des Aliments. Table INRA, Edition Quae : Versailles.

Meffeja F, Njifutié N, Manjeli Y, Tchoumboué J, Tchakounté J. 2007. Digestibilité comparée des rations contenant de la drêche ensilée des brasseries, du tourteau de palmiste ou des coques de cacao chez le porc en croissance finition au Cameroun. Livest. Res. Rur. Dev., 19(5).

Menke KH, Raab L, Salewski A, Steingass H, Fritz D, Schneider W. 1979. The estimation of the digestibility and metabolizable energy content of ruminant feeding stuffs from the gas production when they are incubated with rumen liquor in vitro. J. Agric. Sci. Camb., 93: 217-222.

Menke KH, Steingass H. 1988. Estimation of the energetic value obtained from chemical analysis and in vitro gas production using rumen fluid. Anim. Res. Dev., 28: 7-55.

Moujahed N, Kayouli C, Thewis A, Beckers Y, Rezgui S. 2000. Effects of multinutrient blocks and polyethylene glycol 4000 supplies on intake and digestion by sheep fed Acacia cyanophylla Lindl. foliage-based diets. Animal Feed Science and Technology, 88(3): 219-238.

Mwendia C, Khasataili M. 1990. Molasses blocks for beef cattle. In Utilization of Research Results on Forage and Agricultural By-Product Materials as Animal Feed Resources in Africa Dwozela BH, Said AN, WindemAgenehu A, Kategile JA (eds). Proceedings of the first joint workshop held in Lilongwe, Malawi, 5 - 9 December, 1988, 389 - 403.

Okorondu SI, Akujobi CO, Okorondu JN, Anyado-Nwadike $\quad$ SO. 2013. Antimicrobial activity of the leaf extracts of Moringa oleifera and Jatropha curcas on pathogenic bacteria. Int. J. Biol. Chem. Sci., 7(1): 195-202.

Rabéha C, Brahim H, Aristide L. 2010. Blocs multi nutritionnels à base de rebuts de 
dattes pour ovins dans les zones arides :

1. Caractéristiques physiques et appétibilité. Secheresse, 21: 266-71.

Rivière R. 1991. Manuel d'Alimentation des Ruminants Domestiques en Milieu Tropical. IEMT, Collection Manuel et Précis d'Elevage. La Documentation Française : Paris.

Sansoucy R, Arts G, Preston TR. 1986. Molasses urea blocks as a multinutrient supplement for ruminants. FAO Animal Production and Health Paper: Sugarcane as Feed, 72: 263-278.

Sauvant D, Perez JM, Tran G. 2004 .Tables de Composition et de Valeur Nutritive des Matières Premières Destinées aux Animaux d'Elevage: Porcs, Volailles, Bovins, Ovins, Caprins, Lapins, Chevaux, Poissons. INRA Editions et AFZ : Paris.

Soumana I, Ayssiwede SB, Issa S, Guero, Missohou A. 2016. Effets de la complémentation avec des blocs multi- nutritionnels concentrés à base de Sida cordifolia L., une plante invasive, sur les performnaces zootechnicoéconomiques des antenais Balami à Déréki/Dosso (Niger). ISSR Journal, 15(3): 716-730.

Tabai S. 2009. Essai de fabrication de blocs multi nutritionnels à base de rebuts de dattes, de pédicelles, de paille d'orge et d'urée. Mémoire de fin d'étude en vue de l'obtention du Diplôme d'Ingénieur d'Etat en Sciences Agronomiques, Université KasdiMerbah-Ouargla, Algérie, p.106.

Zogang BF, Boukila B, Sawa C, Tendonkeng F, Tovignon GZ, Pamo ET. 2012. Palatability and physical characteristics of multi-nutrient blocks based on Tithonia diversifolia associated with Brachiaria ruziziensis straw as supplements for Djallonke sheep. Livest. Res. Rur. Dev., 24(3): 41. 